\title{
Timed Up and Go Functional Test
}

National Cancer Institute

\section{Source}

National Cancer Institute. Timed Up and Go Functional Test. NCI Thesaurus. Code

C115795.

A standardized rating scale developed by Mathias et al in 1986 which is used to test basic mobility skills in frail elderly persons and other populations such as people with arthritis, stroke, and vertigo. This functional test measures the time in seconds for a person to stand up from a standard armchair, walk 3 meters, turn, walk back to the chair, and sit down again. Scoring of this test is based on the average of multiple completed and timed trials. 